\title{
Reserves from Controllable Swimming Pool Pumps: Reliability Assessment and Operational Planning
}

\author{
M. Sadegh Modarresi \\ Texas A\&M University \\ sadegh.modarresi@tamu.edu
}

\author{
Le Xie \\ Texas A\&M University \\ le.xie@tamu.edu
}

\author{
Chanan Singh \\ Texas A\&M University \\ singh@ece.tamu.edu
}

\begin{abstract}
This paper introduces a conceptual framework, a capacity assessment method, and a data-driven optimization algorithm to aggregate flexible loads such as in-ground swimming pool pumps for reliable provision of spinning reserves. Enabled by Internet of Things (IoT) technologies, many household loads offer tremendous opportunities for aggregated demand response at wholesale level markets. The spinning reserve market is one that fits well in the context of swimming pool pumps in many regions of the U.S. and around the world (e.g. Texas, California, Florida). This paper offers rigorous treatment of the collective reliability of many pool pumps as firm generation capacity. Based on the reliability assessment, an optimal scheduling of pool pumps is formulated and solved using scenario-based approach. The case study is performed using empirical data from Electric Reliability Council of Texas (ERCOT). Cost-benefit analysis based on a city suggests the potential business viability of the proposed framework.
\end{abstract}

\section{Introduction}

The increasing level of uncertain energy resources in the power grid has been a driving force of research innovation in the past decade around the world. This, compounded by the retirement of many coal-fired fossil fuel generation sources, has introduced substantial challenges in reliable and efficient operation of electric grid. As an example, the Electric Reliability Council of Texas (ERCOT) has 20\% of total generation capacity as wind power and reached the record of the wind share in the total energy production (50\%) on March 23, 2017 [1]. Such change of generation portfolio leads to many changes in both physical and market operations.

To manage the uncertainties at the operating stage, four classes of complementary approaches have been proposed. The first approach is to utilize controllable conventional generating units to compensate the uncertainty. A great body of research has focused on this topic in the past quarter of a century [2], [3]. The main advantage of this approach is that the reserve procured from conventional power plants is firm capacity and there is almost no uncertainty about the capacity of these resources to replace intermittency/contingency in modern power systems. However, it is costly because certain capacity of power plants need to be dedicated to providing the reserve for the system while it can be used for the energy market. Furthermore, since the location of the source of uncertainty and load might be different, deliverability of such reserve, when needed, is another challenge. The second option is holding some capacity of renewables and use it as reserve. With more mature power electronics and forecasting techniques, this option is in the transition from research into practice [4], [5]. The third option is to install energy storage systems to manage uncertainty and enhance the reliability of power systems. Finding the optimal location and storage sizing is one of the major challenges in the third option since capital investment cost for these resources are still high [6], [7]. The fourth option is using the adaptability of load to provide flexibility to the bulk power system. This option is becoming more practical with the increasing availability of two way communication through the Internet. This paper focuses on this option. In particular, we present a framework of aggregating many end-use loads, to provide reliable reserves with little or no impact on consumer's comfort.

There have been many efforts controlling smart appliance inside the houses to provide ancillary service to the grid. These range from appliances that are being used in every day life such as refrigerators [8], to weather dependent appliances like heating, ventilation, and air conditioning (HVAC) systems [9], [10].

The above-mentioned work mainly focuses on gaining benefits from the thermal inertia of refrigerators and HVAC systems. While controlling appliances inside the house for regulation reserve sounds logical (due to zero mean nature of this signal), these resources may not be ideally suitable to provide the spinning reserve to the grid. Spinning reserve deployment signal is not a zero mean signal and if deployed, the duration of its deployment can take from a few minutes to up to two hours [11], [12]. Due to the reliance of these resources on thermal inertia, they are unable to keep providing the spinning reserve for an extended period of time. In [11] the spinning reserve was only activated for a duration of 520 minutes for customers in California. Activation is through a begin-curtailment signal sent to customers. Customers remain curtailed until they receive an end-curtailment signal or their timer operates. Although no complaint from customers is registered in [11], it might not be true in all climate conditions. A study performed in [13] shows that indoor 
temperature can rise 4 degrees Fahrenheit in 5 minutes. Therefore, even if the spinning reserve is deployed for 20 minutes, the household temperature may become noticeably different at the end of the deployment of spinning reserve. It was shown in [11] that with less reliable generators $(95 \%$ individual availability), load resources (with $90 \%$ individual availability) can be a more reliable resource than conventional power plants. Although the message for the given set of availability of power plants and loads is true, the reliability assessment is oversimplified. Since conventional power plants are highly maintained generation resources, their availability will remain constant during their lifetime. However, the reliability of one-way radio communication to a switch can decrease rapidly as time passes. If twoway radio communication is implemented, a discussion on maintaining the reliability is needed as well.

Meyn et al., pioneered the usage of swimming pool pumps as an ancillary service provider [14]-[16]. The design of their work is to provide zero-mean regulation $\{$ up,down reserve to the grid through randomized $\{\mathrm{ON}, \mathrm{OFF}\}$ actuation of pool pumps in real-time. The control strategy is randomized and completely decentralized through mean field limit. The regulation signal is sent every 4 to 5 seconds and each pool pumps takes a sample of this signal every 30 minutes and arranges its behavior based on the number of hours it has been $\{\mathrm{ON}, \mathrm{OFF}\}$ in a stochastic manner. When the number of pools is large enough (one million in the case of Florida), this randomized control provides firm regulation to the grid.

While the work of [14]-[16] addresses the issue of controlling loads to provide continuous AGC services, using the swimming pool pumps in the spinning reserve market might be a better fit for the ancillary service market due to inherent mechanisms of swimming pool pumps. At the beginning of the pumping cycle, pool pumps need a process of sending air out before pumping and circulation of the water begins. During this process, the electricity consumption of the single speed pump is well below the nominal level, e.g. $1.5 \mathrm{~kW}$ average (since it only sends air out) [17]. This process is called priming the pump. The priming process can take between 30 seconds to 30 minutes, with the average of 11 minutes [18]. Therefore system keeps regulation signal active for more than enough time because it does not receive enough response. By the time first-tier pumps finish priming, way more than enough pools received the regulation signal. Therefore an imbalance rebound happens. However, since spinning reserve providers only need to reduce their consumption upon activation, priming will not have an impact on quality of the reserve being gained from the pool pumps.

In this paper, we investigate the benefits of controlling swimming pool pumps $\{\mathrm{ON}, \mathrm{OFF}\}$ status for providing spinning reserve to the wholesale level grid. We show that with proper investment and scheduling, these entities can provide the required level of reserve to the independent system operator (ISO). We show how the capacity of pools can be mapped to the exact quality and quantity from conventional power plants. We also formulate day-ahead market (DAM) participation strategy as an aggregator. Furthermore, a data- driven procurement strategy is proposed. This approach is shown to reduce the realization cost of the aggregator in purchasing energy and reserve from the DAM. Cost-benefit analysis will also be demonstrated.

The key innovation of this paper is suggested as follows:

- a firm reserve capacity is achieved through aggregation of many pool pumps in a rigorous reliability assessment framework.

- an optimal operational planning strategy for aggregators to provide spinning reserve is formulated.

- theoretical performance guarantee is provided for the scenario based operational planning technique.

- the performance guarantee will be shown to only depend on the size of the empirical data being used.

The rest of this paper is organized as follows: Section 2 provides a short background on spinning reserve market, and its procurement structure. Section 3 discusses the way it is possible to design a dominant strategy that benefits customers, aggregators and the ISO. Section 4 introduces the reliability framework of finding the capacity credit of pumps aggregation. Section 5 formulates and solves the operational planning problem from aggregators point of view. Section 6 provides a numerical example of the method introduced. Concluding remarks and future improvements needed for this work are described in Section 7.

\section{Background on Spinning Reserves}

Three major categories of ancillary service market products exist in most deregulated electricity markets: Regulation $\{$ up,down $\}$ reserve, spinning reserve, and non-spinning reserve. Regulation is generally a zero mean signal and providers should be able to respond to this signal within a few seconds. If the disturbance persists, the spinning reserve will replace regulation reserve, within 10 minutes of the first security constrained economic dispatch (SCED) after an event. Non-spinning reserve and (in some markets) supplemental reserve will be deployed if needed as well.

Prices of these products vary based on the system condition and structure of the day ahead market in these systems. The average price of these products in ERCOT from 2016-present is shown in Fig. 1. As can be seen, the spinning reserve is among the highest priced ancillary service product. While the prices always fluctuate across time and regions, this example suggests the potential of tapping into the spinning reserve markets via low-cost and reliable control of end user loads.

Generation adequacy and security in a deregulated electricity market are achieved through communicating with market participants. Market participants are required to provide a share of spinning reserve on the daily operation. They can provide the required share either through their own resources, through bilateral agreements with other market participants, or by participating in the market [19].

Many ISOs in the US send the requirement of spinning reserve share to the market participants before the day ahead market happens. For instance in ERCOT, before the DAM 


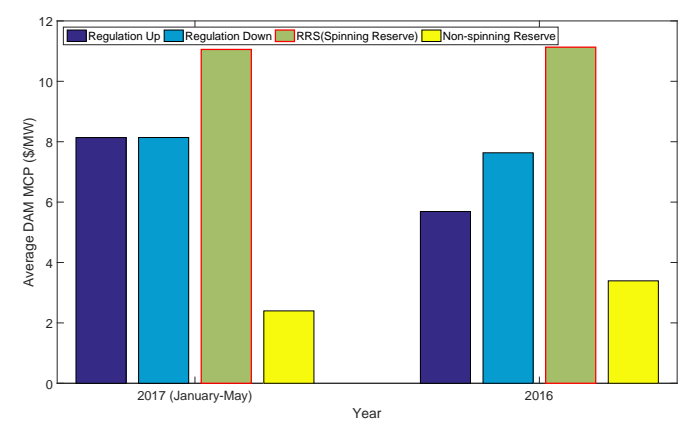

Figure 1. The average prices of different ancillary service products in ERCOT.

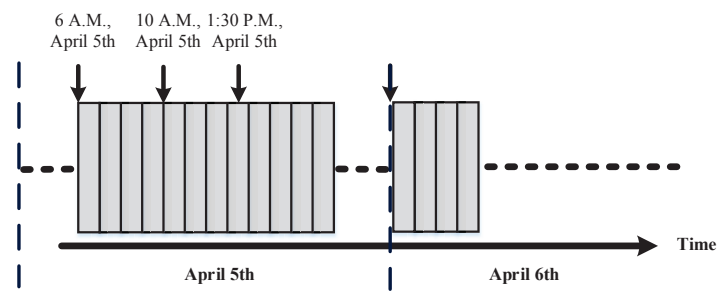

Figure 2. At 6 a.m., ERCOT publishes ancillary service plan for each QSE for the next day. This plan identifies the ancillary service obligation for all QSEs in each hour. At $10 \mathrm{am}$, ERCOT starts the Day-Ahead Market for April 6th based on bids/offers received for energy/reserve for each hour of April 6th. At 1:30 pm, ERCOT closes the day ahead energy and ancillary service market for April 6th and publishes the results.

for each day $d$, at 6 am on day $d-1$ ERCOT assigns part of the ancillary service plan quantity, by service, by an hour, to each load serving entity (LSE) based on its load ratio share during the past seven days. Aggregators participate in the market on behalf of the LSE and they are responsible for providing spinning reserve by any of the three abovementioned methods. Fig. 2 shows the time-line of events in ERCOT. Qualified scheduling entities (QSE) are eligible market participants in the day ahead and real time market in ERCOT.

For an aggregator to qualify the provision of certain ancillary services, several key questions need to be addressed. First, how does ISO certify the resource for ancillary service? Second, what happens at the spinning reserve deployment time? Third, What happens after the recall of deployment? and Fourth, What happens if aggregator failed to provide its spinning reserve share to the market? In the next section, we present the framework of the proposed concept.

\section{Conceptual Analysis of Benefits for Con- sumers, Aggregators and the ISO}

This section offers conceptual analysis of a possible dominant strategy that benefits the three major stakeholders in this process, namely, the end users, the aggregator, and the ISO.

\section{1. "Win" for end users}

Pumps circulate the water through a filter and a chlorinator. Maintaining the chlorine level of the water is an important part of maintaining a healthy pool water. Too much chlorine causes skin and eye irritation. Too low chlorine speeds the formation of algae and causes the water to look cloudy and green. Besides health issues, keeping the pool cleaned is essential to maintain the residential pool permit [20]. Therefore pool owners need to turn their pool pumps $\{\mathrm{ON}\}$ for a certain number of hours every day.

Sunlight, the temperature of water and amount of bacteria in the water have direct correlation to the water's ability to maintain its chlorine level. On a hot sunny Saturday, most swimming pool pumps need to circulate water for their whole cleaning cycle, e.g. for around 12 hours [21]. However, on a cold Monday, not much pool pumping is needed and pool water maintains its chlorine level. If the same full cycle pumping happens for this day, the chlorine content of the pools can reach the unhealthy level.

Keeping the pool water quality within the healthy margin is a burden for the pool owners. Upon questioning some of the pool owners, we found some turn $\{\mathrm{ON}\}$ the pool pump for the entire weekend only, and some invest and buy a timer to turn $\{\mathrm{ON}\}$ the pump for a certain number of hours every day and they adjust the timer based on the season. The first approach can result in the pool water reaching the over-cleaned status and the chlorine level can go above the healthy margin at the end of the weekend. Since water maintains it chlorine level during the cloudy days, fixed number of cleaning hours can also cause over/under cleaning for days with different weather conditions.

Centralizing this control can enhance the comfort of the customers by automatically changing the number of required cleaning hours each day based on the weather forecast. There is also a room to save money for customers with optimal activation of the pumps. Also, since the communication with the smart switches is bi-directional, in the case of a switch failure, the aggregator will be informed to replace the switch.

\section{2. "Win" for aggregators}

Due to health and license concerns mentioned above, swimming pool pumps are must-run loads. However, there is flexibility in the time of the day to run these demands. If aggregators equip the pools in an area with Wi-Fi enabled $\{\mathrm{ON}, \mathrm{OFF}\}$ switches and be able to reliably control them, they can gain benefits by providing a part of their (possible) ancillary service mandate using pools, and potentially participate in the spinning reserve market while controlling a considerable energy consumption of their resources. Capital investment and benefits gained from this investment are key concerns and will be addressed in this paper. It will be shown in Section 6 that given the spinning reserve prices in ERCOT, and only by investment in one city in ERCOT region, aggregators can return their investment capital in less than a year, and gain a profit as high as $\$ 1 \mathrm{M} /$ year afterward. 


\section{3. "Win" for the ISO}

Maximizing social welfare while keeping the system secure is one of the main objectives of ISOs. Normally, a certain capacity of generators is dedicated to providing spinning reserve. It can be shown that reducing the generators' capacity commitment to spinning reserves could increase social welfare. The second advantage of such a reserve comparing to acquiring reserve from conventional generators or big industrial loads is its geographical diversity which is quite desirable during contingencies.

\section{Reliable Reserves from Pool Pumps}

In this section, we present the methodology of firming up reliable reserve (defined in ISO's terms) from the aggregation of many controllable swimming pools. We describe the control method, the reliability assessment method, and the conceptual equivalence to large power plants.

\subsection{Control strategy for pool pumps}

Centralized direct load control is used to control the pool pumps participating in the program. The direct control will be through installing a Wi-Fi enabled $\{\mathrm{ON}, \mathrm{OFF}\}$ switch exclusively for the pool pump. Some examples of commercial $\{\mathrm{ON}, \mathrm{OFF}\}$ switches compatible with the proposed method are [22]-[24], along with many other brands. Their prices typically vary from $\$ 5-\$ 50$ [25] depending on the robustness of embedded software, power rating, and features such as connectivity to other smart home appliances [26].

As discussed in Section 3, pool pumps are located outside the homes and they only need to turn $\{\mathrm{ON}\}$ for a certain number of hours. Therefore, the privacy of customers will not be violated with a centralized control scheme. At the deployment time, after ISO send deployment signal to the aggregator, aggregator updates base points for its resources within a minute. For instance in ERCOT, QSE is required to reach $95 \%$ of this base point within ten minutes. Since communication between the IoT switch and the control center happens in real time every few seconds, 9 minutes is more than enough time to get $95 \%$ required response. In the next subsection, the feasibility is discussed.

\subsection{Capacity credit of pool pumps}

Capacity credit of swimming pool pumps can be defined as the $M W$ capacity equivalent of pools aggregation to 1 $M W$ capacity from a conventional power plant. A reliability assessment of the control and communication network of swimming pool pumps is needed to find the capacity credit of swimming pools. For that purpose, it is essential to know the connectivity structure of the components in the system. The schematic of the system architecture used in this paper is shown in Fig. 3 [27]. In engineering systems, components are connected in parallel, series, meshed or combination of these [28].

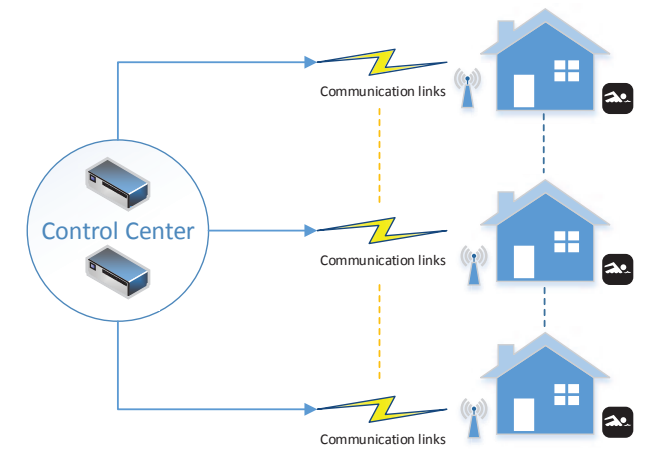

Figure 3. A schematic of system architecture being used.

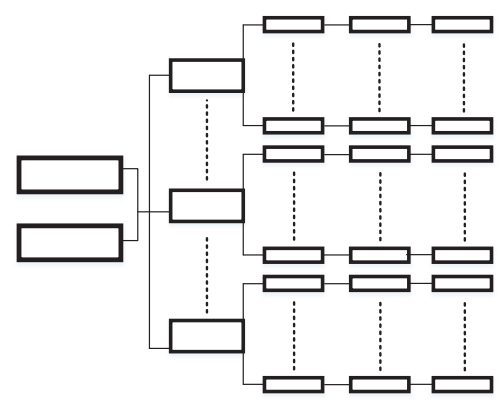

Figure 4. Series/paralleled structure of pool pump control system.

The system being studied normally consists of two control centers (aggregator or QSE), various communication links between the control center and the wireless network of each house, the switch controlling swimming pool pump and the pump itself. Series/parallel structure of mentioned components is illustrated in Fig. 4. Pump, switch, and the home wireless network is in series with each other. A set of homes using the same Internet provider/cable are in parallel with each other. The same series/parallel trend happens till the control center. Since control centers, modern communication networks, and pool pump itself are highly reliable components, without loss of generality, in this paper failures are only considered in-house wireless networks and pool pump switches.

Hazard rate or failure rate of a component is defined as the ratio between the number of components failed in an interval and the average number of components survived in that period [28], [29]. It can be estimated with a replacement test as the ratio of failures to the component hours accumulated. A typical hazard rate curve of an electrical component is shown in Fig. 5. Due to its shape, this figure is normally referred to as bath-tub curve. Bath-tub curve consists of three major parts. In region I, hazard rate decreases as time passes due to a decrease in the possibility of factory errors. In region II, the failure rate is constant, and the component is in its useful life period. In region III, the failure rate starts to increase. It happens when a component exceeds its useful lifetime and should be replaced. More details about bath-tub curve and the useful lifetime of a component can 


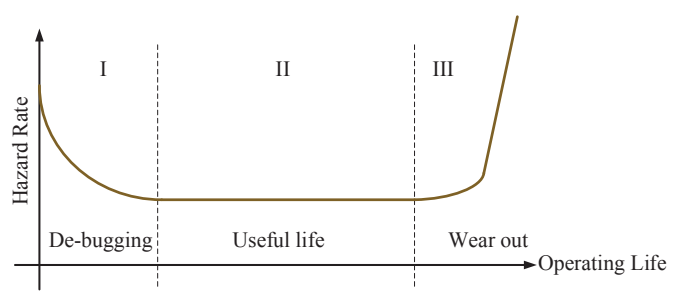

Figure 5. Typical hazard rate of an electrical component as a function of its age.

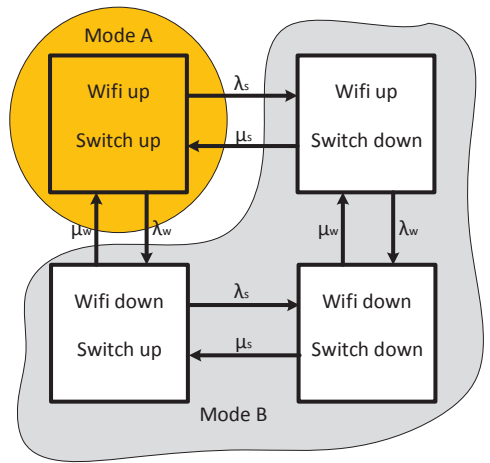

Figure 6. State space diagram for a two-component system: Mode A: Pool pump is accessible for the reserve market, Mode B: Pool pump is not accessible for the reserve market.

be found in [30], [31]. In this paper, components are in their constant hazard rate lifetime, and being replaced afterward. Therefore $\lambda$ and $\mu$ are fixed.

State space diagram of each customer participating in this program is shown in Fig. 6. For a Wi-Fi+modem and an $\{\mathrm{ON}, \mathrm{OFF}\}$ switch with failure and repair rate $\left(\lambda_{w}, \mu_{w}\right.$ and $\lambda_{s}, \mu_{s}$ in Fig. 6 respectively), probability of each pump residing in up mode can be calculated using frequency and duration method showed in [28], [29]. Therefore, accessibility of each pool pump in a house can be calculated as (1). In this paper, the type of all switches an LSE uses to control its customer's pool pumps are assumed to be identical. Also, wireless network in all houses is assumed to have the same failure rate and repair time.

$$
P_{\text {mode }_{A}}=\frac{\mu_{s} \mu_{w}}{\left(\lambda_{s}+\mu_{s}\right)\left(\lambda_{w}+\mu_{w}\right)}
$$

Since each pool is accessible (controllable) with probability $P_{\text {mode }_{A}}$ and it is unavailable with probability $1-$ $P_{\text {mode }_{A}}$, and individual pools are independent of each other, to find the Probability Density Function (PDF) of the total number of available pools, we can use Binomial distribution. For instance using (1) and $\lambda_{w}=\frac{1}{99} f / h r, \mu_{w}=1 r / h r$ and $\lambda_{s}=\frac{1}{9} f / h r, \mu_{s}=1 r / h r$ and $N_{\text {pools }}=34122$, PDF of accessibility of $k$ pools can be drawn as Fig. 7. The probability of getting exactly $k$ pools responsible for the reserve market is $\left(\begin{array}{c}N_{\text {pools }} \\ k\end{array}\right)\left(P_{\text {mode }_{A}}\right)^{k}\left(1-P_{\text {mode }_{A}}\right)^{N_{\text {pools }}-k}$. ERCOT requires at least $95 \%$ of base point in 10 minutes [12]. Assuming that all pool pumps consume the same amount of electricity while on $\{\mathrm{ON}\}$ status, to find the

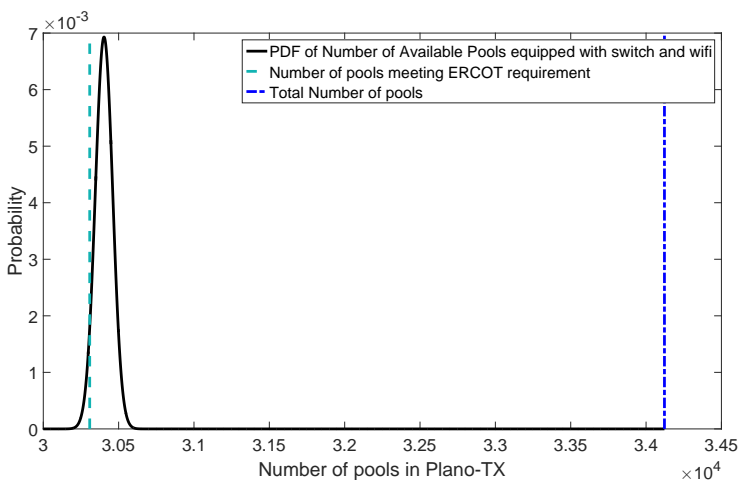

Figure 7. Illustration of qualified pools, and PDF of available pools.

equivalent number of pools, the inverse Binomial probability distribution can be used. This is the level that is qualified for participation/procurement in the ERCOT market. For this example, this level is shown by green dashed line in Fig. 7 .

\section{Scheduling the Pool Pumps for Operational Planning}

Based on the discussion in the previous section, in this section, we discuss how to schedule pools for each hour of the day. From Section 4.2, we have $s r^{\text {pools }}[t]=f(u[t])$, where $u[t]$ is the aggregated $M W$ of pools capacity and $f(u[t])$ maps capacity of pools to their capacity credit, sr pools $[t]$. sr pools $[t]$ should be chosen such that for the entire day it minimizes the cost of aggregator in procuring energy to serve pools and reserve from the DAM when desirable. Therefore, a co-optimization of energy (that is going to serve part of the reserve) and reserve (from the DAM) is needed to find the best operating plan for the aggregator.

Since in this stage a decision is going to be made on the $\{\mathrm{ON}, \mathrm{OFF}\}$ statuses of pool pumps for each hour of the next day, we potentially face with $24 \times N_{\text {pools }}$ integer variables in the optimization problem. However, assuming that all pool pumps consume $1.5 \mathrm{~kW}$, we can use the relaxation method used in [32], and solve for the total $M W$ from pools, knowing that $u[t]=1.5 \times 10^{-3} \sum_{p=1}^{N_{p o o l s}} p_{i}[t]$, where $\left\{p_{i}[t] \in\{0,1\}\right\}$ is the $\{\mathrm{ON}, \mathrm{OFF}\}$ status of individual pool pumps and $N_{\text {pools }}$ is the total number of pools under control. After $u[t]$ for all hours schedules, it will be distributed among the pools.

\subsection{Deterministic bidding approach}

This process happens after aggregator received its obligation in providing spinning reserve for each hour of the next day, $S R^{r e q}[t]$, at 6 am on day $d-1$ in Fig. 2. Also by this time, the number of hours pools need to be cleaned on the next day, $\mathcal{H}_{d}^{r e q}$ is defined based on discussion in Section 3. The deterministic optimization that tells aggregator how to schedule its pool pumps and how much reserve to purchase from the DAM is as (2). $\pi^{E}[t]$ and $\pi^{R}[t]$ in (2a) 
are forecasted DAM energy and reserve prices. $f(u[t])$ is the function maps capacity to capacity credit comes from Section 4.2. It will be shown that for the structure of this problem, it can be written as $f(u[t])=\frac{1}{\mathcal{D}} u[t]$ where $\frac{1}{\mathcal{D}} \leq 1$ is the estimated degrading factor. In case that all components in the system are fully reliable, $\mathcal{D}=1$. (2b) ensures aggregator complies with the ISO requirement. (2c) ensures pools are being cleaned the minimum required number of hours every day. $\mathcal{H}_{d}^{\text {req }}$ is the number of hours each pool pump needs to circulate water per day $d$ and $U^{\text {max }}=1.5 \times 10^{-3} N_{\text {pools }}$ is the maximum $M W$ capacity from swimming pools equipped with required devices. Constrains (2d) ensures the capacity of pools scheduled to be $\{\mathrm{ON}\}$ does not exceeds the maximum capacity of pools combined.

$$
\begin{array}{ll}
\min _{u[t], s r^{D A M}[t]} & \sum_{t=1}^{T=24} \pi^{E}[t] \mathcal{D} u[t]+\pi^{R}[t] s r^{D A M}[t] \\
\text { s.t. } & f(u[t])+s r^{D A M}[t] \geq S R^{r e q}[t] \quad \forall t \\
& \sum_{t=0}^{24} u[t] \geq \mathcal{H}_{d}^{r e q} U^{\text {max }} \\
& 0 \leq u[t] \leq U^{\text {max }} \quad \forall t
\end{array}
$$

We showed the deterministic formulation of our problem. However, since the operational planning stage happens before the DAM, energy and reserve prices are yet to be known and uncertain. Therefore there is a need to manage this uncertainty in the planning problem. In Section 5.2 we discuss our solution for this challenge.

\subsection{Scenario-based optimization: background}

Uncertainty in the price of energy and reserve can cause a noticeable change in the realization of results of (2). In other words, if (2) is solved with $\left\{\pi_{1}^{E}[t], \pi_{1}^{R}[t]\right\}$ and get $\left\{u[t], s r^{D A M}[t]\right\}$, with a perturbed set of prices, $\left\{\pi_{2}^{E}[t], \pi_{2}^{R}[t]\right\},\left\{u[t], s r^{D A M}[t]\right\}$ might not be the solutions to (2). As a response, robust approaches try to solve the problem for the worst realization of uncertainty. They include an uncertainty set for the price and solve for the best strategy as in [33]. However, first, it guarantees the optimality only if realizations of uncertainty occur inside the predefined uncertainty set or dynamically evolving uncertainty set, and second, the level of conservativeness is defined by the length of the uncertainty set. For prices of energy, this length can vary from a negative price (for energy price) to the price cap, based on the Lagrangian multipliers in the economic dispatch problem ISO solves [34].

In recent years, with the proliferation of sensors and computational power, it is becoming increasingly desirable to obtain insights from empirical data. However, conventional sample-based optimization techniques lack the theoretical guarantees of operational risks. More recently, a sample-based optimization approach termed as scenario approach has been proposed [35]. This approach has several desirable features for operational planning decision

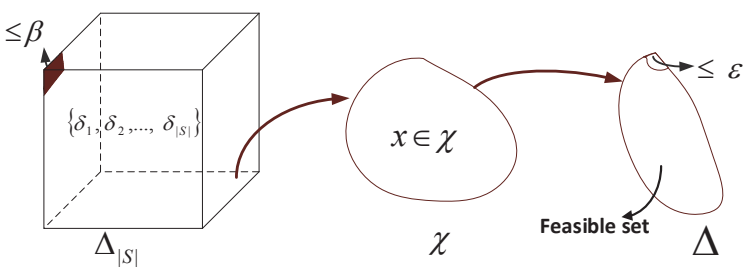

Figure 8 . With the probability of larger than $1-\beta$, the optimal solution, $x^{*}$ is feasible for all constraints in $\Delta$ but at most $\epsilon$ of uncertainties.

making. Two of the most useful features of scenario approach technique are: 1 - It requires only samples from the known/unknown uncertainty set, and the number of these samples are finite and 2- The number of samples needed, defines the robustness of the results. An upper level of risk can be defined based on the number of decision variables $\nu$ and the number of samples available, $|\mathcal{S}|$.

The scenario approach essentially takes the following steps to solve a problem in dimension $\nu$ : step 1, determine the risk tolerance and the level of confidence to the results ( $\epsilon$ and $\beta$ in Fig. 8 respectively); step 2, acquire adequate data $(\mathcal{S}$ in Fig. 8) that guarantees the risk and confidence levels and solve the problem (finding $x$ which minimizes $z(x)$ ). The number of samples for a given level of risk and confidence and $\nu$ can be calculated using Theorem 1.

Definition 1 (Scenario Problem (SP)). $S P_{|\mathcal{S}|}$, for $|\mathcal{S}|$ samples, $\left\{\delta_{1}, \delta_{2}, \ldots, \delta_{|\mathcal{S}|}\right\}$ can be formulated as (3).

$$
\begin{aligned}
S P_{|\mathcal{S}|}= & \min _{x \in \mathbb{R}^{\nu}} c^{T} x \\
& \text { s.t. } \quad f\left(x, \delta_{s}\right) \leq 0,\left\{\delta_{1}, \delta_{2}, \ldots, \delta_{|\mathcal{S}|}\right\} \in \mathcal{S} \subset \Delta
\end{aligned}
$$

Definition 2 (Violation probability $\boldsymbol{V}(\boldsymbol{x})$ ). If $x^{*} \in \mathcal{X}_{\mathcal{S}}$ be the solution for $S P_{|\mathcal{S}|}$, then the $V\left(x^{*}\right)$ can be defined as (4).

$$
V\left(x^{*}\right)=\mathbb{P}\left\{\delta \in \Delta: f\left(x^{*}, \delta\right)>0\right\}
$$

Theorem 1. Given $\epsilon \in(0,1)$ and $\beta \in(0,1)$, and $\nu$ in (3) if

$$
|\mathcal{S}|=\frac{2}{\epsilon}\left(\ln \frac{1}{\beta}+\nu\right)
$$

then $\mathbb{P}\left\{V\left(x^{*}\right) \leq \epsilon\right\} \geq 1-\beta[36]$.

\subsection{Scenario-based bidding approach}

Since constraints in (2) are all deterministic constraints, they are remaining the same in the $(1-\epsilon)$ robust operational planning (scenario-based) formulation. However, the objective function tries to minimize the cost of operational planning for the worst realization of uncertainty among $|\mathcal{S}|$ scenarios. The objective function of our scenario problem is as (6).

$$
\min _{\left\{u[t], s r^{D A M}\right.} \max _{[t]\}\left\{\pi^{E}[t], \pi^{R}[t]\right\} \in \mathcal{S}} \sum_{t=1}^{T=24} \pi^{E}[t] \mathcal{D} u[t]+\pi^{R}[t] s r^{D A M}[t]
$$


Where $\mathcal{S}=\left\{\mathcal{S}_{1}, \mathcal{S}_{2}, \ldots, \mathcal{S}_{|\mathcal{S}|}\right\}$ are energy and reserve empirical samples of $|\mathcal{S}|$ days chosen randomly from the DAM results of previous days. If we rewrite this problem in epigraphic format, we can solve this problem as a linear programming one [35]. Full epigraphic formulation of the problem is as (7). (7b) is the only uncertain constraint in this problem and it will be violated with the probability of at most $\epsilon$. Therefore, the realization cost for the decision made in (7) will not exceed $h$ with $(1-\epsilon) \times(1-\beta)$ confidence. It should be noted that as $\epsilon$ goes to zero, the solution of scenario problem approaches the robust solution which might be too conservative, while $\beta$ can be chosen as a very small number, e.g. $10^{-10}$ without a big impact on the number of required scenarios as shown in (5).

$$
\begin{array}{ll}
\min _{\left\{u[t], s r^{D A M}[t], h\right\}} & h \\
\text { s.t. } & \sum_{t=1}^{T=24} \pi^{E}[t] \mathcal{D} u[t]+\pi^{R}[t] s r^{D A M}[t] \leq h, \\
& f(u[t])+s r^{D A M}[t] \geq S R^{r e q}[t] \quad \forall t \\
& \sum_{t=0}^{24} u[t] \geq \mathcal{H}_{d}^{r e q} U^{\max } \\
& 0 \leq u[t] \leq U^{\text {max }} \quad \forall t
\end{array}
$$

\section{Case Study}

This case study is performed based on data from PlanoTexas. Plano is located near Dallas in North Texas. The percentage of houses having swimming pools in this city is the 10th in the US and highest in Texas. According to [37], 31\% of houses in this city have a swimming pool (Google Earth view over this city can be seen in [38]). Using population and people per household data in [39], the number of swimming pools is estimated as 34122 pools. The assumption in this case study is that all participating pools are the same size, equipped with $1.5 \mathrm{~kW}$ pool pump. Therefore, it gives the aggregator a maximum total capacity of 51.183 MW, 4-12 hours per day. However, based on Subsection 4.2, not all of this capacity is qualified for scheduling. First in 6.1, we discuss reliability assessment of three different investment strategies, then in 6.2 results of the operational planning stage based on formulation in 5.1 and 5.3 will be compared. A discussion on the costbenefit analysis of three investment cases will be performed in 6.3 .

\subsection{Reliability assessment}

According to the discussion in Section 4.2, failures are considered for the wireless network and $\{\mathrm{ON}, \mathrm{OFF}\}$ switches. It is assumed that all houses in this case study affluent enough to have a private pool, already equipped with a Wi-Fi network. Failure rate and repair time of the
Wi-Fi network in these houses are shown in Table 1. Also, it was assumed that Wi-Fi network in these houses being maintained by their owners and its failure rate always resides in region II of Fig. 5.

Three different strategies were considered to invest on the $\{\mathrm{ON}, \mathrm{OFF}\}$ switch installation. A very reliable switch with 10 years useful life and nominal rating of $1.8 \mathrm{~kW}$ (switch type 1) [22]. A reliable switch with the same rating [24] and 5 years nominal life (switch type 2), and a basic switch with a nominal rating of $1.5 \mathrm{~kW}$ [23] and with three years average life in region II of Fig. 5 (switch type 3). It should be emphasized that numbers in Table. 1 are indicative and not factory announced data. Also, a failure in the switch is considered as a failure of the switch software in accurately following the orders from the Wi-Fi network and not the switch hardware itself.

TABLE 1. RELIABILITY PARAMETERS OF COMPONENTS

\begin{tabular}{l|ccc} 
Component & Region II Width (years) & $\lambda$ (failure/hr) & $\mu($ repair/hr) \\
\hline Wi-Fi & N.A. & $\frac{1}{99}$ & 1 \\
Switch Type 1 & 10 & $\frac{1}{999}$ & 1 \\
Switch Type 2 & 5 & $\frac{1}{99}$ & 1 \\
Switch Type 3 & 3 & $\frac{1}{9}$ & 1 \\
\hline
\end{tabular}

Following the procedure in Section 4.2 and using the data in Table 1 for each investment pattern, $P_{\text {mode }_{A}}$ can be calculated as shown in Table 2. The percentage of available pools as a function of the number of pools is as shown in Fig. 9. There will be a steep drop in the percentage of usable pools capacity for the reserve market if the number of pools participating in the program is not big enough (e.g. $<1000$ pools). Capacity credit as a function of participated capacity is shown in Fig. 10. As can be seen, this function can be represented as a linear function, $f_{(x)}=\frac{1}{\mathcal{D}} x$ as was used in the operational planning stage formulation.

For the number of pools in Plano, the estimated $\mathcal{D}$ is available in Table 2. As can be seen, selecting a cheap switch causes a higher $\mathcal{D}$ and in turn higher cost of energy in (2) and lower qualified reserve in (2b). Therefore selecting the right switch will affect the daily cost/benefit of the aggregator.

In the next section, we demonstrate the operational planning stage. To avoid redundant discussions, we fix the switch implemented in the system to the type 3 in this section. In 6.3 , the summary of results using all three approaches are summarized.

TABLE 2. RELIABILITY ASSESSMENT RESULTS

\begin{tabular}{l|ccc} 
Investment & Type 1 & Type 2 & Type 3 \\
\hline$P_{\text {mode }_{A}}$ & $999 \times 99 / 100000$ & $99 \times 99 / 10000$ & $9 \times 99 / 1000$ \\
$\mathcal{D}$ & 1.0121 & 1.0216 & 1.1258
\end{tabular}

\subsection{Bidding the pools into the market}

In this section we show the market participation results. ERCOT spinning reserve requirement used in this paper is 


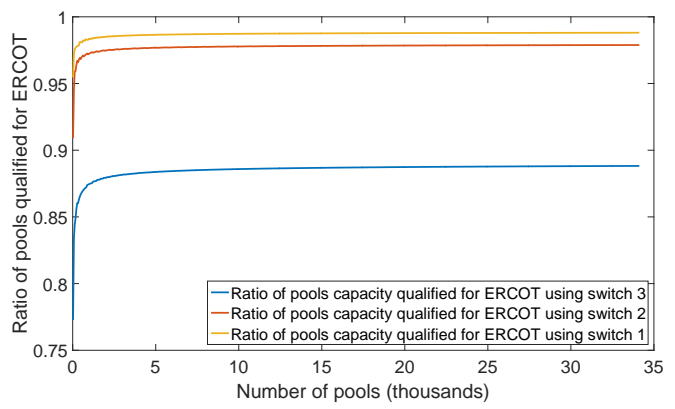

Figure 9. The correlation between the number of participating pools numbers and the ratio of useful spinning reserve for the market:D is very high for small, (e.g. $<1000)$ number of pools.

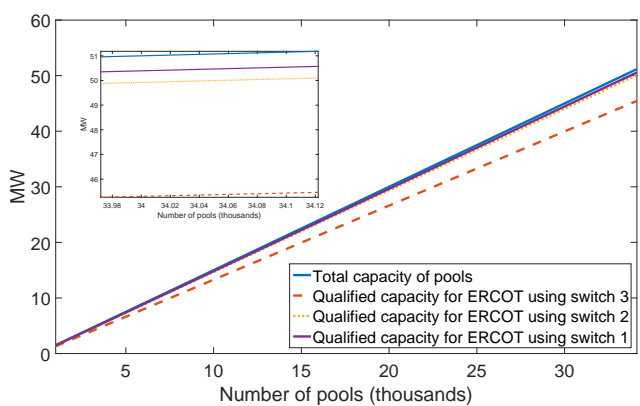

Figure 10. The relationship between the number of participating pools and the reliable provision of spinning reserves.

based on May 27 requirement available in [40], normalized to the population of Plano-TX. As can be seen by yellow dashed lines in Figs. 11,12,13,14, ERCOT reduces the requirement during the day due to low wind forecast and lower uncertainty during these hours. Price samples were extracted for January-April of the past five years. For $\epsilon=0.25$ and $\beta=10^{-3}, 440$ samples we collected randomly to solve (7) based on the requirement in (5). Since the dimension of (7) is always fixed, the number of samples needed is only correlated to the risk tolerance for the results. Another same sized set of samples also were randomly collected to find the realization cost of decision in (7) and (2). Two different pool pumping scenarios considered in this section. One is for the times that potential of algae production is high according to the discussion in Section 3. Therefore there will be a need to pass the entire water of the pool through the chlorinator to increase the chlorine content of the water. The other day is a day that not much filtration is needed.

6.2.1. A hot sunny Saturday. In this scenario, the aggregator sees the forecasts of day-ahead that has a big potential for algae production, because Saturday is going to be a sunny, warm and high pool usage potential. Therefore the decision will be to operate pool pumps for the full cycle, 12 hours. The operational planning results are shown in Figs. 11 and 12. As can be seen, considering the uncertainty of the price of energy and reserve has changed the results. We tested the results using 440 realizations of prices to compare the

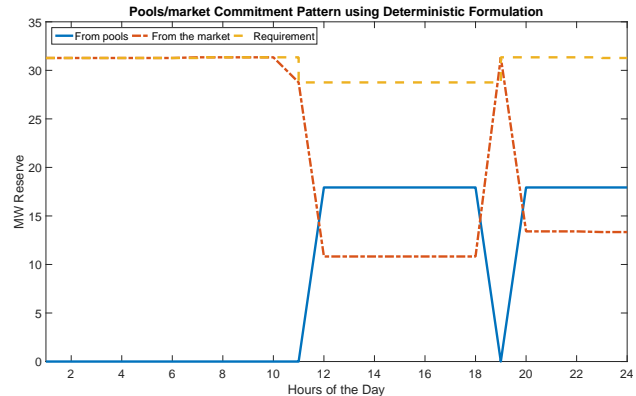

Figure 11. Operational planning results using the deterministic formulation: A hot sunny Saturday.

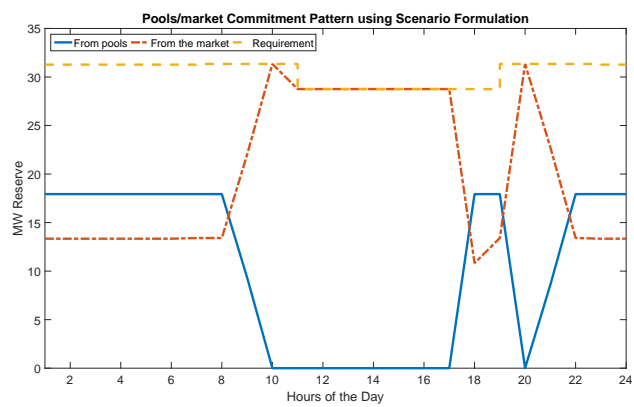

Figure 12. Operational planning results using the scenario approach formulation: A hot sunny Saturday.

results of deterministic and scenario operational planning approach.

Comparing statistical indices on the realization cost of the different approaches, it was observed that the average realization cost has decreased by $7.4 \%$ in the scenario approach formulation. The standard deviation of the realization cost of scenario formulation is $27 \%$ lower than deterministic formulation and the maximum realization cost of scenario formulation is $27 \%$ lower as well.

6.2.2. A cloudy cold Monday. In this scenario, aggregator day-ahead forecast shows a cloudy colder than usual Mondays in Plano. Therefore it decides not to operate its controlled pool pumps for the whole 12 hour cycle. It is due to the fact that the main cause of reduction in the chlorine content of water, bacteria and sunlight, and indirectly water temperature, are at a low level.

Operational planning results can be found in Figs. 13 and 14. Comparing the scenario-based and deterministic approach, it can be observed that although not much pool operation happened, average realization cost has decreased by $7 \%$ in the scenario-based approach. Standard deviation and maximum realization cost have decreased by $16 \%$ and $18 \%$ in the scenario-based approach.

\subsection{Cost-benefit analysis}

The cost-benefit analysis is done by using historical spinning reserve price in ERCOT for the first four months of 


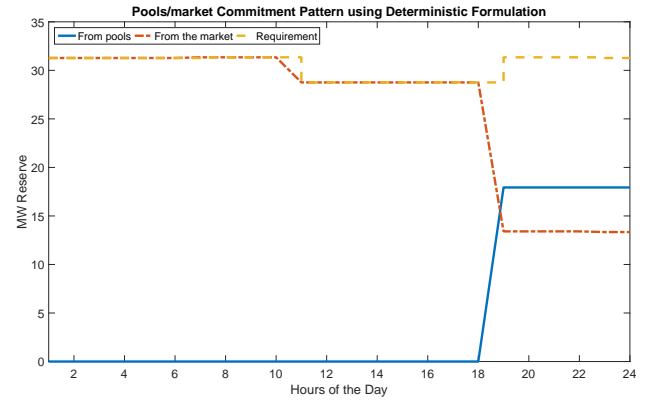

Figure 13. Operational planning results using the deterministic formulation: A cloudy cold Monday.

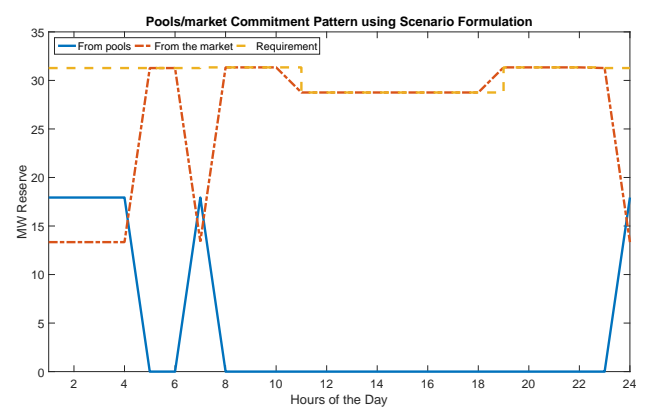

Figure 14. Operational planning results using the scenario approach formulation: A cloudy cold Monday.

2017. Three mentioned switch investment cost is considered along with one time $\$ 1$ million flat upfront investment cost (other than the cost of switches). This cost should cover organizational costs needed for the aggregation strategy mentioned in the paper. The study for all investment strategies was performed over the span of 10 years. Switches are being replaced after their lifetime. Average cleaning of 8 hours per day is assumed. As can be seen in Table 3, the net present value for the cheapest switch is less than other investment strategies due to worse degrading factor and recurrent investment cost over the 10 years span. Switch 1 , however, has the highest net present value, although it has the highest investment cost. Considering the fact that the lifetime of this switch is the highest among all three switches, it leads to the highest net present value for an investment. The results of the net present value analysis in Table 3 are based on 5\% interest rate for the entire 10 years study period. Given the assumption above, type 1 switch is the best investment strategy.

TABLE 3. COST BENEFITS ANALYSIS RESULTS: $\$ 1$ MILLION FLAT UPFRONT ORGANIZATIONAL COST (OTHER THAN THE COST OF SWITCHES) APPLIED TO ALL CASES

\begin{tabular}{l|ccc} 
Investment & Type 1 & Type 2 & Type 3 \\
\hline Cost Per Switch $(\$)$ & 30 & 15 & 5 \\
Annual Profit $(M \$)$ & 1.51 & 1.37 & 1.21 \\
Net Present Value $(M \$)$ & 10.30 & 9.26 & 8.19
\end{tabular}

It should be noted that some other factors might affect the results in Table 3. Factors such as: different average cleaning hours resulting from higher or lower number of days with cold weather for the city under investigation, different reserve prices for regions other than ERCOT, as well as possible installation costs. Future work will consider the impact of the price uncertainty on the performance of the investment.

\section{Concluding Remarks}

We propose a control strategy, reliability assessment and operational planning framework for an aggregator to control the swimming pool pumps and utilize their capacity credit for the provision of spinning reserves in wholesale markets. It is shown that the exact capacity of pools qualified to participate in the market can be found using a reliability assessment over all components that can interrupt the connectivity of the control center and a pool pump. Based on the capacity credit from the pools, an optimal bidding strategy is formulated for the aggregators to participate in the day-ahead reserve markets. By utilizing a scenariobased approach, it is shown that it can save up to $27 \%$ in the realization cost of procurement strategy. A cost-benefit analysis is performed to demonstrate the investment strategy for the aggregators using the ERCOT market as an example.

This paper opens several avenues for future work. On the reliability assessment side, currently, we assume different customers with pools are independent of each other. However as discussed in [41], some factors can cause a dependency between failures of individual customers. Weather conditions such as sudden freezing temperature, and power system condition such as abnormal voltage and abnormal frequency, and cyber attacks [42] are among the reasons that can cause dependency between switches. Modeling of these interdependencies is among our future work for the reliability assessment portion of this paper.

On the operational planning side, a few topics need further research. First, the number of hours pools should remain $\{\mathrm{ON}\}$ before turning $\{\mathrm{OFF}\}$ in this paper is set to be one hour. However, the number of $\{\mathrm{ON}, \mathrm{OFF}\}$ operation per day can have an impact on the customer's cost as well as cleaning performance. Solving the problem in the presence of different pool pumps nominal consumption, and extra procurement of reserve in DAM for possible participation in the real-time energy market using pool pumps capacity is among our future avenues of research.

\section{Acknowledgement}

This work was supported in part by Power Systems Engineering Research Center.

\section{References}

[1] "ERCOT quick facts," (Date last accessed 12-June-2017). [Online]. Available: http://www.ercot.com/content/wcm/lists/114739/ERCOT_ Quick_Facts_4317.pdf

Page 2555 
[2] L. Soder, "Reserve margin planning in a wind-hydro-thermal power system," IEEE Transactions on Power Systems, vol. 8, no. 2, pp. 564-571, May 1993.

[3] L. Xie, P. M. S. Carvalho, L. A. F. M. Ferreira, J. Liu, B. H. Krogh, N. Popli, and M. D. Ilic, "Wind integration in power systems: Operational challenges and possible solutions," Proceedings of the IEEE, vol. 99, no. 1, pp. 214-232, Jan 2011.

[4] M. Hedayati-Mehdiabadi, K. W. Hedman, and J. Zhang, "Reserve policy optimization for scheduling wind energy and reserve," IEEE Transactions on Power Systems, vol. PP, no. 99, pp. 1-1, 2017.

[5] J. B. Cardell and C. L. Anderson, "A flexible dispatch margin for wind integration," IEEE Transactions on Power Systems, vol. 30, no. 3, pp. 1501-1510, 2015.

[6] P. Xiong and C. Singh, "Optimal planning of storage in power systems integrated with wind power generation," IEEE Transactions on Sustainable Energy, vol. 7, no. 1, pp. 232-240, 2016.

[7] Y. Xu and C. Singh, "Power system reliability impact of energy storage integration with intelligent operation strategy," IEEE Transactions on smart grid, vol. 5, no. 2, pp. 1129-1137, 2014.

[8] D. Angeli and P.-A. Kountouriotis, "A stochastic approach to dynamic-demand refrigerator control," IEEE Transactions on control systems technology, vol. 20, no. 3, pp. 581-592, 2012.

[9] E. Vrettos, F. Oldewurtel, and G. Andersson, "Robust energyconstrained frequency reserves from aggregations of commercial buildings," IEEE Transactions on Power Systems, vol. 31, no. 6, pp. 4272-4285, 2016.

[10] J. MacDonald, P. Cappers, D. Callaway, and S. Kiliccote, "Demand response providing ancillary services," Grid-Interop, 2012.

[11] J. H. Eto, J. Nelson-Hoffman, C. Torres, S. Hirth, B. Yinger, J. Kueck, B. Kirby, C. Bernier, R. Wright, A. Barat et al., "Demand response spinning reserve demonstration," Lawrence Berkeley National Laboratory, 2007.

[12] "Current protocols - nodal," (Date last accessed 12-June-2017). [Online]. Available: http://www.ercot.com/mktrules/nprotocols/current

[13] W. Jewell, "Residential energy efficiency and electric demand response," in 2016 49th Hawaii International Conference on System Sciences (HICSS), Jan 2016, pp. 2435-2444.

[14] S. Meyn, P. Barooah, A. Bui, and J. Ehren, "Ancillary service to the grid from deferrable loads: The case for intelligent pool pumps in florida," in 52nd IEEE Conference on Decision and Control, Dec 2013, pp. 6946-6953.

[15] P. Barooah, A. Buic, and S. Meyn, "Spectral decomposition of demand-side flexibility for reliable ancillary services in a smart grid," in 2015 48th Hawaii International Conference on System Sciences (HICSS), Jan 2015, pp. 2700-2709.

[16] S. P. Meyn, P. Barooah, A. Bušić, Y. Chen, and J. Ehren, "Ancillary service to the grid using intelligent deferrable loads," IEEE Transactions on Automatic Control, vol. 60, no. 11, pp. 2847-2862, 2015.

[17] "Programming suggestions for centrifugal pumps," (Date last accessed 12-June-2017). [Online]. Available: http://www.wentec. com/unipower/miscellaneous/pdf/centrifugalpump.pdf

[18] "Intelliflo Vf pump installation and users guide," (Date last accessed 12-June-2017). [Online]. Available: http://www.pentairpool.com

[19] "Nodal 101," (Date last accessed 12-June-2017). [Online]. Available: http://www.ercot.com/services/training/course/109518\#materials

[20] "Residential pools," (Date last accessed 12-June-2017). [Online]. Available: http://www.houstontx.gov/health/Environmental/ residentialpools.html

[21] "What causes algae problems?" (Date last accessed 12-June-2017). [Online]. Available: http://www.poolcenter.com/algae

[22] "Wemo mini smart plug," (Date last accessed 12-June-2017). [Online]. Available: http://www.belkin.com/us/F7C063-Belkin/p/PF7C063/
[23] "Sonoff - wifi wireless smart switch," (Date last accessed 12-June2017). [Online]. Available: https://www.itead.cc/smart-home/sonoffwifi-wireless-switch.html

[24] "Smart wifi meter plug 31," (Date last accessed 12-June-2017). [Online]. Available: http://www.orvibo.com/en/product/47.html

[25] "smart wifi switch," (Date last accessed 12-June-2017). [Online]. Available: https://goo.gl/J0T2WU

[26] “Amazon alexa," (Date last accessed 12-June-2017). [Online]. Available: https://developer.amazon.com/alexa

[27] F. Kamyab, M. Amini, S. Sheykhha, M. Hasanpour, and M. M. Jalali, "Demand response program in smart grid using supply function bidding mechanism," IEEE Transactions on Smart Grid, vol. 7, no. 3, pp. 1277-1284, May 2016.

[28] R. Billinton and R. N. Allan, Reliability evaluation of engineering systems. Plenum Press, 1992.

[29] C. Singh and R. Billinton, System reliability, modelling and evaluation. Hutchinson London, 1977, vol. 769.

[30] M. Bebbington, C.-D. Lai, and R. Zitikis, "Useful periods for lifetime distributions with bathtub shaped hazard rate functions," IEEE Transactions on Reliability, vol. 55, no. 2, pp. 245-251, 2006.

[31] H. Pham and C.-D. Lai, "On recent generalizations of the weibull distribution," IEEE transactions on reliability, vol. 56, no. 3, pp. 454458, 2007.

[32] A. Halder, X. Geng, P. R. Kumar, and L. Xie, "Architecture and algorithms for privacy preserving thermal inertial load management by a load serving entity," IEEE Transactions on Power Systems, vol. PP, no. 99, pp. 1-1, 2017.

[33] A. A. Thatte and L. Xie, "A robust model predictive control approach to coordinating wind and storage for joint energy balancing and frequency regulation services," in 2015 IEEE Power Energy Society General Meeting, July 2015, pp. 1-5.

[34] A. A. Thatte, X. A. Sun, and L. Xie, "Robust optimization based economic dispatch for managing system ramp requirement," in 2014 47th Hawaii International Conference on System Sciences (HICSS), Jan 2014, pp. 2344-2352.

[35] A. Care, S. Garatti, and M. C. Campi, "Scenario min-max optimization and the risk of empirical costs," SIAM Journal on Optimization, vol. 25, no. 4, pp. 2061-2080, 2015.

[36] A. Carè, S. Garatti, and M. C. Campi, "Fast-fast algorithm for the scenario technique," Operations Research, vol. 62, no. 3, pp. 662$671,2014$.

[37] Y. Pan, "Top 10 cities for making a splash in an amazing home swimming pool," (Date last accessed 12-June-2017). [Online]. Available: http://www.realtor.com/news/trends/top-10cities-for-pool-loving-homeowners/

[38] "Google earth view of plano residential area," (Date last accessed 12-June-2017). [Online]. Available: https://goo.gl/eU8zOP

[39] "United states census bureau," (Date last accessed 12-June-2017). [Online]. Available: https://www.census.gov/

[40] "Day-ahead market," (Date last accessed 12-June-2017). [Online]. Available: http://www.ercot.com/mktinfo/dam

[41] M. Papic and et. al, "Research on common-mode and dependent (cmd) outage events in power systems: A review," IEEE Transactions on Power Systems, vol. 32, no. 2, pp. 1528-1536, March 2017.

[42] C. Singh and A. Sprintson, "Reliability assurance of cyber-physical power systems," in IEEE PES General Meeting, July 2010, pp. 1-6. 